\title{
Newton's experimental proofs ${ }^{\&}$
}

\section{(Las pruebas experimentales de Newton)}

\author{
Timm LAMPERT* \\ Humboldt University Berlin
}

\begin{abstract}
Newton claims that his theorems in the Opticks are derived from experiments alone. The paper explains this dictum by relating Newton's proof method to an iconic conception of proof as opposed to a symbolic one. Theorems are not derived from hypotheses; instead properties of light are identified by experimental properties based on rules of inductive reasoning.
\end{abstract}

KEYWORDS: Iconic Proof; Newton; Experimentum Crucis; Diagrammatic Reasoning.

RESUMEN: Newton afirma que sus teoremas en la Óptica se derivan exclusivamente de experimentos. El artículo explica esta afirmación relacionando el método de prueba de Newton con una concepción icónica de las pruebas, en contraposición a una concepción simbólica. Los teoremas no se derivarian de hipótesis, sino que las propiedades de la luz se identifican a partir de propiedades experimentales basadas en reglas de razonamiento inductivo.

PALABRAS CLAVE: Prueba icónica; Newton; Experimentum Crucis; Razonamiento diagramático.

\& The paper profited considerably from concise comments from an anonymous referee concerning, among other topics, modern proof theory, the use and interpretation of the term experimentum crucis, the role of diagrams and further relevant literature. This is a revised version of talks held at Berlin, Rostock, Siegen and Stourbridge. I am grateful to the audiences for the related discussions.

* Correspondence to: Timm Lampert. Department of Philosophy, Humboldt University Berlin, Unter den Linden 6 (D-10099 Berlin) - lampertt@ staff.hu-berlin.de - https://orcid.org/0000-0003-3912-7933

How to cite: Lampert, Timm (2021). «Newton's experimental proofs»; Theoria. An International Journal for Theory, History and Foundations of Science, 36(2), 261-283. (https://doi.org/10.1387/theoria.21155). 


\section{Introduction}

Newton's claim to provide experimental proofs is often criticized. It is argued that his proofs are based on hypotheses and not inferred from the experiments alone. This criticism, however, applies a hypothetico-deductive analysis to Newton's experimental reasoning. Such an analysis is not consistent with Newton's own understanding of his proof method. The following reconstruction of Newton's proof method is intended to do justice to his understanding by applying the conception of iconic proofs to Newton's proofs by experiment. The main purpose of this analysis is to explain Newton's dictum that the experiment alone serves as the source of evidence from which his theorems are derived. After drawing a general distinction between symbolic and iconic proofs and illustrating this distinction by means of Euclidean proofs and Aristotelian syllogisms, I will apply this distinction to Newton's experimental proofs and analyze Newton's proof of the heterogeneity of sunlight by his experimentum crucis as an iconic proof. Finally, I will show that this experiment and its underlying method remain prominent in Newton's Opticks.

\section{Symbolic vs. Iconic Proofs}

Iconic proofs are distinct from symbolic proofs. The constituents of symbolic proofs are sentences, while the constituents of iconic proofs are icons. According to Peirce, an icon represents what it represents because it resembles what it represents. By contrast, a symbol symbolizes what it symbolizes merely by convention. This difference can be utilized within proofs: In symbolic proofs, the properties in question are not identified by means of the properties of the symbols (sentences) used in those proofs. Sentences are proven by logical deduction from axioms. By contrast, iconic proofs prove the properties in question by means of the properties of the icons used in those proofs; the properties of the icons are used as identity criteria for the properties in question.

- Symbolic conception of proof: A proof is a logical deduction of a sentence (the proven theorem) from other sentences (the axioms).

- Iconic conception of proof: A proof is a rule-governed construction of an icon that provides the proof criteria for what is in question.

The icons providing the relevant proof criteria according to the iconic conception may be diagrams such as the diagrams used in Euclid's geometric proofs, or they may be Venn diagrams, Peirce's existential graphs or Wittgenstein's ab-diagrams, which are used in iconic proof procedures of first-order logic. Euclid's diagrams represent relevant geometric properties; Venn diagrams, Peirce's graphs and Wittgenstein's ab-diagrams represent relevant logical properties (Lampert, 2017, 2018, 2020).

Symbolic as well as iconic proofs can be understood in either a subjective or an objective sense. In the first sense, a proof is intended to convince an audience; in the second, a proof is a stepwise, rule-governed construction that answers a question by relating it to something given. A symbolic proof persuades an audience given that the audience accepts the axioms on which it rests. An iconic proof persuades an audience given that the audience shares the adopted criteria for evidence. A symbolic proof relates a theorem to given axioms by deducing the theorem from the axioms through the stepwise application of deductive rules. An iconic proof relates a property in question to a property of an icon through the application of nondeductive (e.g., ge- 
ometric, diagrammatic or inductive) rules. An icon is a source of objective evidence, meaning that the property in question can be identified by a property of the icon.

Since the emergence of mathematical logic at the end of the 19th century, a symbolic conception of proofs dominated both, modern proof theory as well as the rational reconstruction of prominent proofs in the history of science. Induced by the so-called 'iconic turn', this, however, changed by the work of the recent decades regarding both, the systematic investigation of what proofs are as well as the understanding of historical proofs. Principles of symbolic and iconic proofs are only spelled out rigorously within a modern understanding of formal calculi, be it in terms of rules to deduce theorems or rules to generate icons. The formal elaboration of a proof conception helps to make explicit or to work out rigorously implicit principles of historical proofs. In doing so, it is not claimed that the founder of the proofs were in the possession of a formal and explicit understanding of their proof methods; it is merely presumed that historical proofs are based on underlying proof ideas. To make them explicit, one must draw distinctions between different proof conceptions in the first place. To understand the strugle regarding proofs in the history of sciences better, we need clearcut conceptions of proofs that help to make implicit principles of historical proofs explicit. In the following, I will first illustrate differences between a symbolic and an iconic conception of proof by providing alternative reconstructions of geometrical proofs, illustrated by Euclid's proof of his first proposition, and of logical proofs, illustrated by Aristotle's syllogism Barbara; then, I will draw analogies to the analysis of Newton's experimental proof in his experimentum crucis and argue that experiments serve as icons in Newton's experimental proofs.

\subsection{Symbolic vs. Iconic Analysis of Euclidean Proofs}

In his first proposition, Euclid proves how to construct an equilateral rectangle. This proof is often criticized to be incomplete. This criticism, however, rests on a reconstruction in terms of a symbolic conception of proof.

Euclid's proof refers to the following rules that he establishes prior to his proofs (Heath, 1968, pp. 153-155, discussed in detail on pp. 183, 187, 195, 199, and 222):

D15: A circle is a plane figure contained by one line such that all the straight lines falling upon it from one point among those lying within the figure are equal to one another.

D20: Of trilateral figures, an equilateral triangle is that which has its three sides equal, $[\ldots]$.

P1: [...] to draw a straight line from any point to any point.

P3: To describe a circle with any center and distance.

CN1: Things which are equal to the same thing are also equal to one another.

In illustrating the symbolic conception of proof, I abstain from a detailed logical analysis of Euclid's proof. Such an analysis would be enormously intricate and extend beyond Euclid's text. In particular, a full logical formalization of a valid deductive formal proof would require further axioms that are neither among Euclid's presumptions nor mentioned in the proof of proposition $1 .{ }^{1}$ Thus, it would be even more evident that Euclid's proof is

1 Cf., e.g., (Tarski, 1959); (Beeson, 2019, pp. 35-36); and http://geocoq.github.io/GeoCoq/. (Dal Magro et al., 2019), referring to forthcoming work by Lassalle Casanave, also note differences between Euclidean proofs by diagrams and Hilbert's axiomatic proof conception. 
incomplete according to a logical analysis. Instead of a detailed logical reconstruction, I will be satisfied with analyzing Euclid's proof in the form of a sequence of sentences, as provided in Euclid's text and based on his postulates, definitions and common notions. This suffices to show that the judgment that Euclid's proof is incomplete depends on the analysis of his proof as a sequence of sentences. Table 1 provides a simplified symbolic reconstruction of Euclid's proof.

\section{Table 1. Symbolic Reconstruction of Euclid's Proof}

\begin{tabular}{l|c|l|l}
\hline \multicolumn{4}{c}{ Problem: On a given finite straight line to construct an equilateral triangle } \\
\hline Division & No. & \multicolumn{1}{c}{ Sentence } & \multicolumn{1}{c}{ Rule } \\
\hline Setting out & 1 & Let the straight line $A B$ exist. & Assumption \\
\hline Construction & 2 & The circle $B C D$ with center $A$ and radius $A B$ exists. & P3 \\
& 3 & The circle $A C E$ with center $B$ and radius $B A$ exists. & P3 \\
\hline ? & 4 & The two circles $B C D$ and $A C E$ intersect at point C. & $?$ \\
\hline Construction & 5 & The straight lines $C A$ and $C B$ exist. & P1 \\
\hline & 6 & $A C=A B$ & $1,2,4,5$, Def. 15 \\
Core Proof & 7 & $B C=B A$ & $1,3,4,5$, Def. 15 \\
& 8 & $C A=A B=B C$ & 6,7, CN1 \\
\hline Conclusion & 9 & The triangle $A B C$ is equilateral given $A B$. & 8, Def. 20 \\
\hline
\end{tabular}

As has often been noted, this proof has a gap on line $4 .^{2}$ Furthermore, sentences within a symbolic proof must assert propositions. Euclid's text, however, presents instructions that explain how to construct a diagram. According to a symbolic conception of proof, P1 and P3 must be interpreted as assumptions of existence, although they in fact refer to actions. Thus, the analysis of Euclid's proof as a symbolic proof neither does justice to the wording of Euclid's text nor yields a compelling symbolic proof.

The sentences in Euclid's proof do not state propositions. Instead, they either are instructions for generating a diagram or point to the relevant properties of the generated diagram that prove the geometric properties in question. According to the symbolic conception of proof, the diagram accompanying the text in (Heath, 1968, p. 241) merely illustrates the proof; it is neither part of the proof nor does it have any probative force. According to an iconic reconstruction of Euclid's proof, however, the proof consists not of the sentences but of the diagrams to which the sentences refer. Table 2 analyzes the proof step by step and relates the sentences to the corresponding diagrams. ${ }^{3}$ The sentences are reproduced almost exactly from (Heath, 1968, p. 241f.).

2 Cf., e.g., (Heath, 1968, p. 242):

It is a commonplace that Euclid has no right to assume [...] that the two circles will meet in a point $C$. For a discussion, cf., for example, (Giaquinto, 2011); (Beeson, 2019, p. 2).

3 For similar reconstructions, cf. (Byrne, 1847, p. 1); (Miller, 2001, p. 2). That diagrams play an essential role in Euclid's proofs has been accepted as standard since the work of, e.g., (Manders, 2008; Mumma, 2012). 
Table 2. Iconic Reconstruction of Euclid's Proof

\begin{tabular}{|c|c|c|c|c|}
\hline \multicolumn{5}{|c|}{ Problem: On a given finite straight line to construct an equilateral triangle } \\
\hline Division & No. & Sentence & Diagram & Rule \\
\hline Setting out & 1 & Let $A B$ be the given finite straight line. & $A \longrightarrow \longrightarrow$ & P1 \\
\hline \multirow{3}{*}{$\begin{array}{l}\text { Construction } \\
\text { of the diagram }\end{array}$} & 2 & $\begin{array}{l}\text { With center } A \text { and distance } A B \text {, let } \\
\text { the circle } B C D \text { be described. }\end{array}$ & & P3 \\
\hline & 3 & $\begin{array}{l}\text { With center } B \text { and distance } B A \text {, let the } \\
\text { circle } A C E \text { be described. }\end{array}$ & & P3 \\
\hline & $4 / 5$ & $\begin{array}{l}\text { From the point } C \text {, in which the circles } \\
\text { cut one another, to the points } A \text { and } \\
B \text {, let the straight lines } C A \text { and } C B \\
\text { be joined. }\end{array}$ & & $2 \times \mathrm{P} 1$ \\
\hline \multirow{3}{*}{$\begin{array}{l}\text { Identification } \\
\text { of geometric } \\
\text { properties }\end{array}$} & 6 & $\begin{array}{l}\text { Since the point } A \text { is the center of the } \\
\text { circle } C D B, A C \text { is equal to } A B .\end{array}$ & & D15 \\
\hline & 7 & $\begin{array}{l}\text { Since the point } B \text { is the center of the } \\
\text { circle } C A E, B C \text { is equal to } A B \text {. }\end{array}$ & & D15 \\
\hline & 8 & $\begin{array}{l}\text { Since } A C \text { and } C B \text { are equal to } A B, C \\
A \text { is also equal to } C B .\end{array}$ & & CN1 \\
\hline Solution & 9 & $\begin{array}{l}\text { Since the three straight lines } C A, A B \\
\text { and } B C \text { are equal to one another, the } \\
\text { triangle } A B C \text { is equilateral. }\end{array}$ & & $\mathrm{D} 20$ \\
\hline
\end{tabular}


According to an iconic understanding of Euclid's proof, the postulates refer to the drawing of diagrams and the relation of the drawn figures to geometric figures. P1 and P3 refer to actions: drawing a line using a ruler and drawing a circle using a compass. However, a line that is drawn in this way is not exactly straight, is not without breadth and is not continuous. Similar caveats apply to the described circle: not every mark that constitutes the described line will lie at exactly the same distance from the center. Nevertheless, P1 and P3 postulate that the drawings have the geometric properties of the figures they represent. Although the drawn figures do not exactly resemble the geometric figures they represent and although they have a specific magnitude, it is postulated that their resemblance to those geometric figures is sufficiently exact to allow them to play the role of the geometric figures themselves and that their relevant properties represent the geometric properties of the represented geometric figures in general within the proof. The drawn figures are not instances of generalities that can only be stated through propositions; instead, per the postulates, they are representatives (paradigms) of arbitrary figures such that whatever can be shown by referring to the relevant properties of those representatives may serve as proof of the properties of the geometric figures. Consequently, the properties of diagrams that follow from the rule-guided construction of such representatives, such as the intersection of drawn circles, may serve as criteria for proof; no further proof is needed that the drawn circles in fact intersect because this fact can be read off from the diagram on the basis of its rules of construction. Consequently, the iconic reconstruction of Euclid's proof has no gap that must be filled by stipulating the intersection point $C$.

Thus, to close the gap in Euclid's proof, no further assumption is needed; only a different analysis, based on a different conception of proof, is necessary. The probative force of the iconic proof originates from the relevant properties of the constructed diagram. Euclid's calculus is a special calculus for constructing specific diagrams and reading off their geometric properties; it is not a logical, topic-neutral, deductive calculus for deducing propositions from axioms. One might question the use of non-deductive postulates, definitions and common notions to establish the role of diagrams in representing the properties of geometric objects within Euclidean geometry. However, in doing so, one does not identify a gap within Euclid's proofs. Instead, one rejects the Euclidean calculus itself and, consequently, the praxis and application of Euclidean proofs using a ruler and compass.

\subsection{Symbolic vs. Iconic Analysis of Syllogisms}

Properties that can be advantageously read off from diagrams without needing to be justified by further statements or inferences are known as "free rides" or "observational advantages" in the literature; see, e.g., (Shimojima et al., 1996; Stapleton et al., 2016, 2017). This concept is relevant not only for the analysis of Euclid's proof but also in the case of logical proofs. In the case of an analysis of syllogisms as sequences of sentences, for example, one needs to introduce and justify deductive inference rules in order to identify correct syllogisms. For example, in the case of the syllogism Barbara, no property of the premises can serve as a criterion based on which to infer the conclusion. Instead, inference rules are needed. For brevity, I simply assume one generalized rule $\mathrm{MPP}_{\forall}$ in Table 3 . This rule can be proven in a calculus of natural deduction through universal quantifier elimination, the rule of assumption, modus ponendo ponens (MPP), conditional proof, and universal quantifier introduction. The deductive rules of a logical calculus, in turn, are in need of some 
further meta-logical justification, which is usually provided by semantics showing that truth is preserved in the case of sentences expressing propositions.

Table 3. Symbolic Proof of the Syllogism Barbara

\begin{tabular}{c|c|c|c}
\hline \multicolumn{3}{c}{ Problem: To prove that the syllogism Barbara is a valid } \\
\hline \multicolumn{1}{c|}{ Division } & No. & Sentence & Rule \\
\hline \multirow{2}{*}{ Premises } & 1 & $\forall x(S x \rightarrow M x)$ & Assumption 1 \\
\cline { 2 - 4 } & 2 & $\forall x(M x \rightarrow P x)$ & Assumption 2 \\
\hline Conclusion & 3 & $\forall x(S x \rightarrow P x)$ & $2,1, \mathrm{MPP}_{\forall}$ \\
\hline
\end{tabular}

According to an iconic representation of the syllogism Barbara as a Venn diagram, however, the mere representation of statements of the form $\forall x(F x \rightarrow G x)$ is sufficient to show that the conclusion follows from the premises. In Venn diagrams, the extensions of concepts and their relations are represented by circles and their possible intersections, and statements of the form $\forall x(F x \rightarrow G x)$ are represented by marking the area that must be empty for such a statement to be satisfied. Let us use $\vec{T}$ to denote the rule for representing statements of the form $\forall x(F x \rightarrow G x)$ and $\overleftarrow{T}$ to denote the rule for reading off statements of that form. Table 4 illustrates that the iconic reconstruction of the syllogism Barbara allows one to read off the conclusion from the representation of the premises. The conclusion comes as a "free ride" from the representation of the premises. There is no need for a further meta-logical justification given the rules to decide logical properties by Venn-Diagrams. Understanding an iconic proof depends on recognizing some aspect of a representation rather than on inferring some conclusion from a set of given premises. Again, one might prefer the symbolic conception of logical proofs in terms of deductive calculi and their semantic justification to an iconic conception of a logical calculus based on non-deductive rules to generate icons and deciding logical properties in terms of icons. This, however, is a dispute on the principles of proof conceptions and does not show that iconic proofs of syllogisms are inconsistent, incorrect or incomplete.

The examples above show that the analysis of a proof and its probative force differs significantly between the symbolic and iconic conceptions of proof. In the following, I will demonstrate that this distinction also applies to Newton's experimental proofs.

In the case of Newton's proof of the heterogeneity of sunlight, the experimentum crucis provides the relevant criterion for proof by representing properties of light. In this sense, the experiment is an icon that is utilized within an iconic proof. Newton also uses diagrams to depict his experiments. These diagrams play a role similar to that of the textual descriptions of his experiments or the textual explanations in Euclid's proof: they explain the construction of icons and point out their relevant properties.

In a proper understanding of iconic proofs, it is claimed neither that such proofs are self-evident nor that they are not guided by rules. It must be clearly specified which properties are merely arbitrary properties of the specific representation used in a particular proof and which ones are relevant properties to which the proof refers. As for any proof, one needs to understand the rules that govern such a proof. It is not simply any perceivable ob- 
jects or their properties that may serve as evidence; instead, only perceivable objects that are generated according to certain rules may serve as sources of evidence, and only perceivable properties that are identified according to certain rules may serve as criteria for proof. Iconic representations gain probative force within a framework of such rules. This holds not only for Euclid's proofs and for iconic proof procedures in logic but also for Newton's experimental proofs, as I will illustrate in the following.

Table 4. Iconic Proof of the Syllogism Barbara

\begin{tabular}{|c|c|l|l|l|}
\hline \multicolumn{5}{|c|}{ Problem: To prove that the syllogism Barbara is a valid. } \\
\hline Division & No. & \multicolumn{1}{|c|}{ Sentence } \\
\hline $\begin{array}{l}\text { Translate } \\
\forall x(S x \rightarrow M x) \\
\text { (Premise } 1) .\end{array}$ \\
\hline of the diagram
\end{tabular}

\section{Rules for Experimental Proofs}

At first glance, Newton appears to apply the axiomatic method in his experimental proofs. In his Opticks, he presents definitions and axioms of geometrical optics prior to his proofs of theorems. The appearance of an axiomatic proof method, however, is misleading. Newton's proofs instead are "proofs by experiment". Theorems are inferred directly from experiments that are described by means of text or diagrams. Newton does not explain how he 
infers the theorems from the experiments. He does not refer to single definitions or axioms to justify the steps of his proofs.

Moreover, the definitions and axioms of Newton's Opticks are presumptions of geometrical optics that ground his descriptions of his experiments. This becomes particularly clear from his diagrams of prismatic experiments; see, for example, Figure 1 on p. 273 of this paper. These diagrams sketch the paths of rays starting from a source of light and ending at color patches. The way in which the propagation of light rays is drawn is based on the presumption of Newton's definitions of light rays (Definition 1), their refrangibility (Definition 2) and their reflexibility (Definition 3) as well as the definitions and axioms regarding the geometrical descriptions of refraction and reflection (Definitions 4-7, Axioms 1-8) and their relation to colors (Definition 8). Thus, Newton's definitions and axioms serve as rules for geometrically constructing and interpreting his experiments. Without the theoretical concept of a ray of light and fundamental geometric laws concerning such rays, it is not even possible to pose questions of geometrical optics or to design and interpret experiments to answer those questions.

In this respect, Newton's definitions and axioms serve a purpose with regard to the relationship between experiments and physical entities that is similar to the purpose served by Euclid's postulates with respect to the relationship between diagrams and geometric entities: they establish how experiments in optics are to be designed and physically interpreted such that the properties of an experiment (e.g., color patches on a screen detecting "a beam of light") represent the properties of a physical entity (e.g., rays of light). Similar to the textual descriptions in Euclid's proofs, Newton's diagrams of an experiment show and explain how to construct and interpret the experiment, which is the source of evidence for identifying properties of physical entities. In doing so, Newton's diagrams and textual descriptions both make implicit use of the established definitions and axioms. These rules make it possible to link an experiment with the propagation of light rays in the experimental design.

\section{Table 5. Analogies Between Different Iconic Proofs}

\begin{tabular}{c|c|c}
\hline Icon & Explanation & Properties \\
\hline Euclidean diagram & text & geometrical \\
\hline Venn diagram & text & logical \\
\hline Newtonian experiment & diagram + text & physical \\
\hline
\end{tabular}

The properties of the drawn lines representing rays of light in Newton's diagrams refer to the geometric properties of the rays of light they represent. It is the geometric properties of the rays that are identified by the paths the lines follow, from a source of light through certain media to color patches. Because of this focus on geometric properties, Newton's concept of a ray of light does not imply any assumptions regarding a theory of light (such as that light consists of waves or corpuscles), nor does he attribute colors to rays; rays of light induce colors, but they themselves are not colored (Newton, 1730, pp. 108-109). However, for simplicity, Newton allows one to speak "grossly" (ibid) of rays as being colored. In the same sense, I will use "colored rays" in the diagrams below (see Table 6 on p. 275) to rep- 
resent rays that would induce certain colors if they were to be separated and detected by a screen or the eye.

In analogy to Euclid, I summarize Newton's presumption regarding the geometrical interpretation of optical experiments in the form of the following postulate:

P: To describe the paths of light rays from a light source to patches of color through refracting and reflecting media by means of straight lines.

Newton always emphatically rejects the presupposition of hypotheses and the subsequent use of experiments to eliminate alternative hypotheses in order to indirectly derive a hypothesis. By contrast, he purports to derive his theorems "positively \& directly" (Turnbull, 1959, pp. 96-97) from experiments. He calls his method of proof "induction" (Newton, 1730 , p. 404). Newton's experimental proofs rely on principles of causal or, even more generally, inductive reasoning, such as the principle of causation and other principles of simplicity, such as determinism, continuity, least action and Newton's rules of reasoning in experimental philosophy. This is why Newton regards his experimental proofs as inductive derivations, as opposed to truth-preserving deductive inferences, which he calls "demonstration".

For the analysis of Newton's experimentum crucis, we will need only the following two "rules of induction":

IR1: Any difference is caused by another difference.

IR2: Effects of the same kind, if possible, have the same cause.

IR1 is Newton's version of the principle of causality (Turnbull, 1960, p. 256-257), quoted on p. 274 below. IR2 is Newton's second rule of reasoning in experimental philosophy (Newton, 1962, p. 398). I will discuss the application of these two principles below. For now, it is sufficient to see that Newton applies rules of inductive reasoning to infer causal statements about physical entities from experiments.

\section{Early Modern Proof Theory}

Against the background of early modern proof theories, Newton was an advocate of the ancient geometrical proof method, as opposed to Aristotelian syllogisms in modern natural philosophy and to algebraic proofs, as invented by Descartes, in early modern mathematics (Guicciardini, 2016). He admired the geometrical method for at least two reasons: (i) for not starting with general axioms or first principles without experimental evidence and (ii) for being anchored in physical reality. (i) places the geometrical proof method in opposition to syllogisms, which derive conclusions from axioms, and (ii) places the geometrical proof method in opposition to algebraic proofs, which make use of a formalism that gives rise to artificial problems of interpretation when proving physical properties (Guicciardini, 2016 , p. 408). Newton admired ancient geometry as a paradigm for applying proof methods that provide the evidence needed for proving properties in geometry and physics. His preference for the geometrical proof method fits well with an iconic conception of experimental proofs in geometrical optics. 
Newton explicitly compared his method of experimental proof with a third distinction commonly invoked in early modern proof theories, namely, the distinction between analysis and synthesis, see the final passage of the Opticks, (Newton, 1730, p. 404-405); see also MS Add. 3970, f., quoted in (Shapiro, 2004, p. 196). He was familiar with this distinction as part of the ancient geometrical proof method ${ }^{4}$ and criticized Descartes' use of this distinction in the algebraic proof method. According to Descartes, analysis consists of reducing a geometrical problem to algebra (or, more precisely, to a polynomial equation), while synthesis consists of building up a geometrical construction from algebra (Guicciardini, 2016, p. 402). According to Newton, however, such a geometrical construction stands for itself. Thus, he saw no need to relate geometrical constructions to algebraic criteria. In particular, he did not associate synthesis with geometrical construction on the basis of some more basic algebraic form.

Many historians interpret analysis as a heuristic step prior to proof, while only synthesis provides the proof itself, e.g. (Guicciardini, 2016, p. 405). However, there is no clearcut evidence in the ancient sources for this rather modern way of distinguishing a context of discovery from the context of justification. More importantly, this way of interpreting the distinction does not fit with Newton's understanding of the relation between the two approaches. When Newton says that "through analysis they [i.e., the 'ancients'] discovered propositions, and through synthesis they demonstrated them once found" (Newton, 1962 , p. 415), this does not mean that he identifies "proof" and "demonstration" such that discovering a proposition is not an essential part of a proof. Instead, Newton reserves the term "demonstration" for the deduction performed once a solution or proposition is found. However, it is "analysis [. . . ] that renders them [i.e., propositions] absolutely certain and so, because of their certainty, worthy to be admitted into geometry" (hitherto). Analysis provides certainty because it provides the evidence on which a proof relies. In Euclid's proofs, the method of analysis used to solve a problem consists of geometrical construction (cf. steps 2 to 4 in Table 2), while synthesis is used to show that the geometrical construction thus obtained indeed solves the problem (cf. steps 6 to 8 in Table 2); the method of analysis provides the criteria for identifying the properties in question and, thus, precedes the method of synthesis within a Euclidean proof. Reducing a Euclidean proof to "the synthetic method of proof" goes hand in hand with regarding Euclid's proof method as the first paradigm of the "axiomatic and deductive" proof method, which is the only one that "achieves true scientific" proofs (Guicciardini, 2016, p. 308). This understanding neither does justice to Euclid nor helps to explain Newton's proof theory.

How to interpret the ancient distinction between analysis and synthesis within the geometrical proof method as well as Newton's understanding of it is controversial. In the following, I will concern myself only with the application of this distinction to Newton's ex-

The distinction between analysis and synthesis can be traced back to Plato and Aristotle (Menn, 2002) and can also be found in Euclid XIII. The relevant passage, however, seems to have been inserted into Euclid's Elements in 60 AD by Heron (Heath, 1968, p. 138). (Ihmig, 2004, section 4), argues that Newton refers to the much discussed locus classicus from Pappus (Pappus, 1986, p. 82f.), and (Heath, 1968 , p. 138-139). For an overview of the debate on the primary source of Newton's distinction between analysis and synthesis, see (Ducheyne, 2005, p. 218 and footnotes 8 to 14). For the relation between Newton's proof method and the ancient distinction between analysis and synthesis, see, among others, (Hinitkka et al., 1974, chapter IX); (Guerlac, 1973); (Ihmig, 2004). 
perimental proofs. Plato, Aristotle, Euclid and Pappus all characterize the crucial difference between analysis and synthesis by characterizing analysis as the process proceeding from the sought to the admitted, whereas synthesis proceeds in the reverse direction, from the admitted to the sought. (Ducheyne, 2005, p. 219) rightly points out that Newton does no more than refer to this general and uncontroversial idea of the mathematical tradition in his application of the analysis/synthesis distinction to experimental proofs (Newton, 1730, p. 404). In (i) identifying what is admitted with effects and what is sought with their causes and (ii) identifying analysis with induction and synthesis with deduction, he goes beyond the mathematical tradition and applies it to experimental philosophy. In doing so, Newton contradicts Hooke, who identified synthesis with the inductive and affirmative search for causes from effects, while he considered analysis to be conjectural and negative in explaining effects from causes (Hooke, 1705, p. 330); (Hamou, 2018, p. 59, footnote 31). By contrast, according to Newton, analysis consists of affirmative, non-hypothetical reasoning as the relevant first part of an experimental proof, which establishes causes prior to the presentation of any explanation of phenomena by their causes. If one insists on providing evidence for concluding causes, there is no other way than "the arguing from Experiments [...] by Induction" (Newton, 1730, p. 404).

Newton also applies the dictum that analysis proceeds synthesis to the composition of the first book of the Opticks:

Of this Method I gave an instance in the first book of these Opticks, investigating first by Analysis the original differences of the rays in respect of refrangibility reflexibility $\&$ colour $\&$ then from these <differences considered as $>$ principles compounding explications of the colours made by Prisms, the colours of the Rainbow \& those of natural body. ${ }^{5}$

This statement makes evident (i) that the method of analysis constitutes experimental proof and (ii) that the experimental proofs of the initial propositions of the Opticks are based on this method. Newton first establishes the general causes of refraction, reflection and color (namely, the various properties of light rays) in Part I of Book 1 before he then explains the effects of color in various specific circumstances (prisms, rainbows, and natural bodies) through the method of synthesis (mainly in Part II of Book 1).

According to Newton, analysis is related to two aspects of experimental proofs: (i) "making experiments" and (ii) "drawing general conclusions from them by induction" (Newton, 1730, p. 404). The reasons obtained through analysis are therefore twofold: (i) evidence (ratio cognoscendi) and (ii) causes (ratio essendi). The first is obtained by conducting an experiment, and the second are derived via induction based on the relevant experimental data. Newton applies the term "analysis" to experimental proofs in which causes are inductively inferred from effects produced in experiments that are deliberately designed to allow causes to be inferred. In synthesis, by contrast, causal relations are presumed to explain observed experimental phenomena. Any causal explanation is therefore based on proving a causal relation. The crucial point is that Newton's method of proof does not start from possible causal hypotheses or axioms that might then be related to experimental phenomena in order to eliminate hypotheses. Instead, an experimental proof

510 MS Add. 3970, f. 242v, quoted from (Shapiro, 2004, p. 193); see also (Shapiro, 2004, p. 189); (Newton, 1730, p. 405). 
based on analysis consists of establishing positive evidence that allows one to infer causes through inductive reasoning.

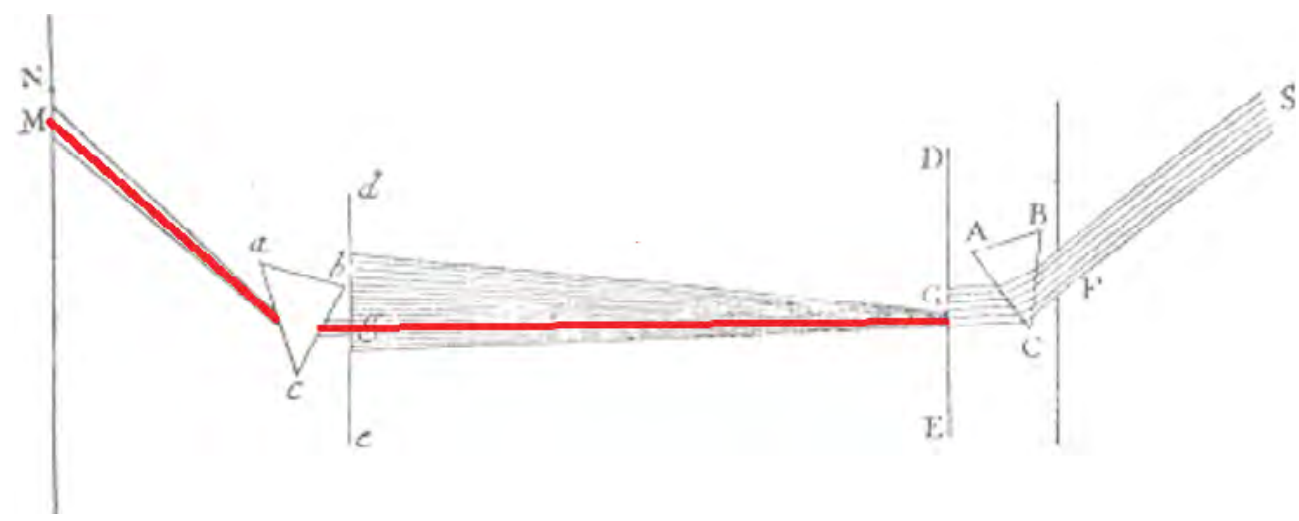

Figure 1

Newton's Experimentum Crucis, from (Newton, 1730, Figure 18)

Newton's application of analysis in experimental proofs can be compared to Euclid's geometric proofs. Just as Euclid's geometric proofs are based on the construction of geometrical diagrams to serve as the source of evidence for identifying the geometric relations in question, Newton's experimental proofs are based on experiments that serve as the source of evidence for identifying the causal relations in question. Just as Euclid's diagrams possess the relevant properties that serve as proof criteria, Newton's experiments provide positive criteria for causal inference. Moreover, just as the understanding of Euclid's diagrams depends on (i) the construction of diagrams to enable the solution of a problem and (ii) the identification of the relevant properties of the diagrams to solve the problem in question, an understanding of one of Newton's experiments depends on (i) a description of the experiment in the context of a causal problem and (ii) the solution to that problem in reference to the relevant properties of the experiment. In this respect, experiments serve as icons in Newton's proofs of geometrical optics. Just as diagrams serve as icons in Euclid's proofs, allowing the properties of geometrical entities to be identified from the properties of the corresponding icons, experiments enable the identification of properties of light from the very properties of the experiments themselves in Newton's proofs. In the following, I will illustrate this by means of Newton's famous experimentum crucis; see (Newton, 1671/2, p. 3078-3079), and Figure 1.

\section{Experimentum Crucis}

With his experimentum crucis (see Figure 1), Newton wishes to elucidate the cause of the oblong form of the spectrum produced when sunlight passes through a prism in the case that the angle of incidence and the angle of emergence are equal (Newton, 1671/2, p. 3076). By rotating the first prism around its axis, Newton moves the spectrum cast on 
the second board in front of the second prism up and down (cf. the two diagrams on line 1 of Table 6, which, in contrast to Figure 1, present two significantly different situations for comparison). To see that the experimentum crucis makes it possible to compare differences under homogeneous conditions, one must understand that the angles of incidence of rays originating from different parts of that spectrum and passing through the second prism are, roughly speaking, identical. These angles are almost identical because of the rather small holes in the boards behind the first and in front of the second prism and the rather long distance between the holes. However, although their incidence angles and all other external conditions are homogeneous, different rays passing through the second prism refract differently behind the second prism: the red spot $M$ on the wall, which is produced by rays originating from the red lower part of the spectrum on the board in front of the second prism, is below the blue spot $N$ on the wall, which is produced by rays originating from the blue upper part of the spectrum. It is the realization of different perceivable effects (the locations of $M$ and $N$ on the wall) under homogeneous conditions that proves that the cause of this difference must be (internal) differences in the properties of the light (cf. the two figures on line 2 of Table 6, which identify the relevant aspects of the dijference test). Such an inference from effects to their causes is based on the principle of causality ("any variation (or difference) must have a cause"; see IR1, p. 10) and presumes conditions of homogeneity. Newton is well aware of this kind of causal reasoning enabled by the design of his experiment, as is clear from a letter he wrote to Lucas, quoted from (Turnbull, 1960, p. 256-257, the brackets are part of the quoted text):

In ye External causes you name there was no difference. The incidence of ye rays ye specific nature of ye Glass ye Prisma figure, \&c were the same in both cases, $\&$ therefore could not cause ye difference: [it being absurd to attribute the variation of an effect to unvaried causes.] All things remained ye same in both cases but ye rays, $\&$ therefore there was nothing but ye difference of their Nature to cause ye difference of their refraction.

The relevant property of the experimentum crucis that justifies Newton's causal inference is the fact that all external factors are identical, yet the positions of the spots on the wall differ. This justifies the identification of internal differences in the rays of light as the cause of the different refraction behaviors behind the second prism, under the presumptions of the principle of causation and the description of the experiment according to Newton's definitions and axioms of geometrical optics.

Newton generalizes his inference that proves the different refrangibility of the light rays passing through the second prism to infer the heterogeneity of the sunlight that passes through the first prism and gives rise to the oblong spectrum on the second board in front of the second prism. This generalization is based on his second rule of reasoning in experimental philosophy: to identical effects (e.g. differences in refraction), one must assign, as far as possible (= as long as no conflict with experimental evidence arises), identical causes (e.g. differences in the properties of the light); see (Newton, 1962, p. 398), and IR2 on p. 270. Clearly, such a rule is not deductive. It is a rule that is needed if criteria for proof are to be drawn from experiments: it allows one to generalize causal inferences drawn from difference tests as long as no other experimental evidence is available.

Table 6 summarizes Newton's "proof by experiment". This proof is "iconic" in the sense that the properties of the experiment (different positions of color patches) represent 
the properties of physical entities (different properties of light rays). From this perspective, Newton's experiments are "iconic representations" that allow one to infer causes from effects. This does not imply that the effects and causes resemble each other visually, in the manner of a portrait and a face. As in geometry, the entities that are represented are abstract (not perceivable). Instead, in the case of iconic representations, resemblance means that (relevant) differences between representations represent differences in what is represented. The presumption of such a resemblance is not a necessary or logical truth, but it is a necessary condition for iconic proofs.

Table 6. Iconic Analysis of Newton's Experimental Proof






\section{Critique of Hypothetico-deductive Analysis}

Newton's experimental proof of the heterogeneity of sunlight by means of his experimentum crucis is often misunderstood as an example of deductive reasoning from alternative hypotheses, in which the experimentum crucis is used to decide among alternative hypotheses. Table 7 presents a simplified version of such an analysis that rests on (Sabra, 1981, p. 249-250) and (Thompson, 1995, p. 8-9).

Table 7. Symbolic Analysis of Newton's Experimental Proof

\begin{tabular}{l|c|l|c}
\hline \multicolumn{1}{c}{ Division } & No. & \multicolumn{1}{c}{ Sentence } & Rule \\
\hline Hypotheses & 1 & $\begin{array}{l}\text { Either light rays are modified by prisms or sunlight is het- } \\
\text { erogeneous. }\end{array}$ & Assumption \\
\hline Experimental fact & 2 & The light rays are not modified by the second prism. & Assumption \\
\hline Conclusion & 3 & Sunlight is heterogeneous. & 1,2, Disj. Syll. \\
\hline
\end{tabular}

On this basis, indirect reasoning by eliminating a hypothesis based on experimental data raises at least two problems: (i) alternative hypotheses may have been overlooked, and (ii) any hypothesis may be made compatible with the experimental data by means of auxiliary assumptions. For example, in the case of the above symbolic analysis, one might concede that the light rays passing through the second prism are not modified by the second prism because the patches $\mathrm{M}$ and $\mathrm{N}$ on the wall are circular (and monochromatic). However, one may still argue that the rays from the sun passing through the first prism are modified by the first prism. Thus, the hypothesis that light rays are modified by prisms is amended to the hypothesis that some light rays (e.g., white light from the sun) are modified by prisms (Sabra, 1981, p. 250).

A modern understanding of crucial experiments in the sense of experiments for deciding among alternative hypotheses does not do justice to Newton. The term "experimentum crucis" was not established in Newton's time and must not be identified with a part of the hypothetico-deductive method as was done later, from the 19th century onward (Hamou, 2018). The term was used first by Boyle in his Defence against Linus in 1662 and second by Hooke in his Micrographia in 1665, both of which adopt Bacon's notion of an "instantia crucis" (Anstey et al., 2008, p. 112). (Hamou, 2018; Schwartz, 2017) have recently argued convincingly that Bacon's notion and his examples of instantiae crucis cannot be reduced to the hypothetico-deductive understanding of eliminating hypotheses by means of negative evidence. Although some of Bacon's examples support such eliminative reasoning (e.g., the tide case), others are used to infer causes positively and directly by means of a difference test, as in the flame-in-a-flame case (Schwartz, 2017, p. 139). Ultimately, then, instantiae crucis can serve a twofold function: to eliminate explanations and to select and affirm explanations. The first function fits well with the hypothetico-deductive method, while the latter fits with the inductive Baconian methodology. 
Boyle and Hooke use the eliminative function of the term experimentum crucis, which is predominant in the later hypothetico-deductive tradition (Hamou, 2018, section 3). Similar to his contradiction of Hooke's application of the analysis/synthesis distinction to experimental philosophy (see above, p. 12), however, Newton also contradicts Hooke's understanding of the experimentum crucis by emphasizing its positive and direct function. He agrees with Hooke that an experimentum crucis serves for a "search after the true cause" (Hooke, 1665, p. 54); (Newton, 1730, p. 3079). He disagrees with Hooke, however, in claiming that the method for concluding the true cause consists of induction and, thus, is a method of analysis.

This does not mean that Newton's experimentum crucis cannot be used in the negative sense to eliminate alternative causal explanations. In fact, in a letter to Pardies, Newton argues en passant against modificationism in a way that is similar to the above hypothetico-deductive reconstruction of the argument of the experimentum crucis (Turnbull, 1959, p. 167); (Cohen, 1958, p. 107-108 for a translation), see Table 7 for the argument. However, Newton does not use this argument to prove his own theory by elimination, nor does he present this kind of argument in his publications; see the following section. Most importantly, he attributes a decisive probative force to the experimentum crucis because it enables a direct causal inference, while he ignores its negative function when arguing how it proves the heterogeneity of sunlight. The modern understanding of an experimentum crucis does not acknowledge that Newton does not derive theorems from sentences by applying logical rules but rather derives theorems from experiments by means of principles of induction. Just as Euclid's definitions and common notions allow geometric properties to be identified as proof criteria, Newton's inductive reasoning allows experimental properties to be identified as criteria for causal judgments.

\section{The First Six Experiments in the Opticks}

(Jalobeanu, 2014) argues that Newton's arrangement of the sequence leading up to his experimentum crucis in his New Theory from 1671/72 has features of Baconian experientia literata: Newton starts with a surprising experiment that gives rise to a causal question not yet answered by any established theory (the one-prism experiment showing the unexpected elongation of the spectrum), then proceeds to experiments that eliminate certain possible causes (e.g., irregularity of the prism, different incidences of the sun rays or the existence of curved rays), and finally presents his experimentum crucis to decide the matter by proving the "true cause" of the effect in question. (Hamou, 2018, p. 62), rightly points out that it makes no sense to analyze Newton's experimentum crucis as eliminating a rival hypothesis since (i) no rival theory existed to explain the new effect and (ii) the experimentum crucis is distinguished from the previous experiments that serve an eliminative function. The sequence of experiments culminates in the experimentum crucis, which provides a positive answer to the initial question.

In the following, I will show that the sequence of experiments in the Opticks leading up to the experimentum crucis follows a similar narrative that culminates in the experimentum crucis. Newton's experimentum crucis is presented as experiment six among ten total experiments that are related to proposition one and two of the Opticks. Proposition two states the heterogeneity of sunlight and is preceded by the first proposition, which states that light 
rays that differ in color also differ in refrangibility. This first proposition is proven by two experiments. From the sequence of six experiments leading up to the experimentum crucis, it may seem as though the experimentum crucis does not, in fact, play a prominent role in the Opticks and also does not serve as a prominent example of an experimental proof according to Newton. In this section, I will show that the opposite is true.

In Newton's first experiment, a half-red and half-blue paper is viewed through a prism; see Figure 2. The blue half (DG) suffers greater refraction than the red half (FE). This experiment can be viewed as a subjective version of the difference test at the second prism in the experimentum crucis. Since, roughly speaking, all conditions are the same except the colors on the sheet of paper, it must be the light rays originating from the differently colored areas of the paper that cause the difference in refraction.

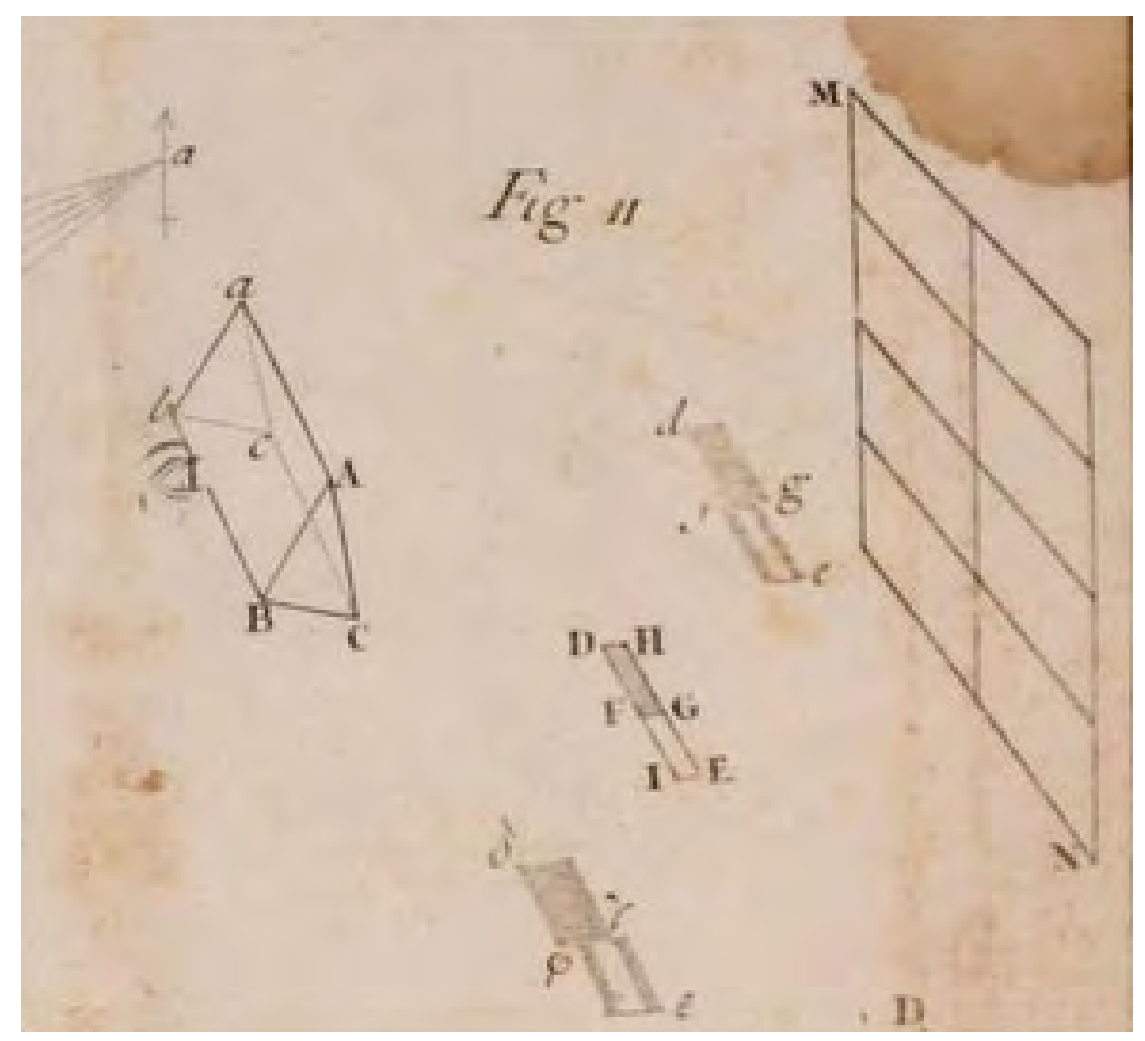

Figure 2

Newton's First Experiment, from (Newton, 1730, Figure 11)

However, it is not spectral lights in a dark chamber but rather painted colors on a black paper that are refracted in this first experiment. This results in colored edges that Newton does not mention. Thus, the phenomenon (the difference in refraction) is not as clear-cut as in the case of the refraction behind the second prism in the experimentum crucis. This 
may be the reason why Newton explicitly states in the Opticks that his experimentum crucis proves not only proposition two but also proposition one; see the quote below on p. 281 . However, from the methodological point of view, these differences with respect to the experimentum crucis only raise doubt as to whether the assumption of homogeneity is fully satisfied in Newton's first experiment. They do not cause one to question the crucial role that difference tests play in Newton's experimental reasoning.

The same argument applies to the second experiment. It is an objective version of the first experiment. In contrast to the first, it takes place in a dark chamber and involves a white sheet of paper. In contrast to both the first experiment and the experimentum crucis, a lens is used, and the source of light is not the sun but a candle. Since the colors are not spectral but rather originate from a sheet of paper, the phenomena are again not as clearcut as in the case of the refraction behind the second prism in the experimentum crucis. However, from the methodological perspective, the intention of the second experiment is also to create a difference test. In both experiments, it is shown (or purported to show) that it must be the light rays causing the appearance of different colors that also cause the differences in refraction because, roughly speaking, all other conditions remain the same.

The first two experiments in the Opticks present simplified and less clear-cut versions of a difference situation similar to the second prism in the experimentum crucis. Creating difference situations through experiments is crucial for inferring causes from effects, as is done in analysis by applying inductive reasoning.

Newton starts the sequence of experiments subsequent to proposition two with two one-prism experiments (experiments three and four). The first is identical to the first part of the experimentum crucis and is the experiment with which he starts his experientia literata in his New Theory of $1671 / 72$. In this experiment, a spectrum is projected onto the wall of a dark chamber. The second is a subjective version of this experiment. It seems surprising that Newton starts his "proofs by experiment" of proposition two with an experiment to which he originally did not attribute any probative force but which he merely used to raise the question of the cause of the surprising effect of the elongation of the spectrum (Newton, 1671/2, p. 3076-3078). However, Newton deliberately does not state that his first experiment proves the heterogeneity of sunlight; rather, he says only that it "agrees with his first proposition" (Newton, 1730, p. 27). Furthermore, he emphasizes that the two experiments do not answer the question of the reason for the different refraction behaviors (Newton, 1730, p. 28):

So then, by these Experiments it appears, that in equal Incidences there is a considerable inequality of Refractions. But whence this inequality arises, whether it be that some of the incident rays are refracted more, and others less, [...], or that one and the same ray is by refraction disturbed, shatter'd, delated and as it were split and spread into many diverging rays, as Grimaldo supposes, does not yet appear by those Experiments, but will appear by those that follow.

From this statement, it is evident that Newton does not regard the single experiments following proposition two as sufficient proofs. Instead, they are part of a sequence arranged to direct the course of investigation towards the experiment crucis, which finally answers the causal question raised by the preceding experiments.

The two one-prism experiments (experiments three and four) are followed by two two-prism experiments (experiments five and six). In experiment five, Newton places a 
second prism immediately after the first in a rotated position such that in the first prism, the rays are refracted upwards, while in the second prism, they are reflected to the side; see "Fig. 14" in Figure 3. Newton observes that the breadth is not increased, but the superior (violet) part again suffers greater refraction than the inferior part after the second refraction; see "Fig. 15" in Figure 3. This experiment is similar to the second experiment in the New Theory, which eliminates irregularities of the prism as a possible cause. It is more akin to what is called an "experimentum crucis" according to hypothetico-deductive analysis. It falsifies one of the two alternative explanations mentioned in the above quote (Newton, 1730, p. 30):

If the rays were dilated [...] so that they should not any longer go in single lines to single points, [...] the second refraction [...] would compose the four square image $\pi \Gamma$. But the event is otherwise. The image PT was not made broader by the refraction of the second Prism.



Figure 3

Newton's Fifth Experiment, from (Newton, 1730, Figures 14 and 15)

Thus, one may indirectly conclude that the cause of the different refraction behaviors is an inherent difference in the rays correlated with the different colors, as has already been sugested by the first experiment (see Figure 2 on p. 18 above). However, Newton neither draws this indirect conclusion nor claims to have proven proposition two before he presents his experimentum crucis. This experiment is experiment six in the Opticks, and it marks the center point of his sequence of experiments following propositions one and two. It combines an objective and spectral version of the difference test from experiment 
one with experiment three (the first subsequent to proposition two) and presents the positive evidence for both propositions one and two (Newton, 1730, p. 38, emphasis mine):

I found that the light which being most refracted in the first Prism did go to the blue end of the image [i.e., the spectrum on the board in front of the second prism-T.L.], was again more refracted in the second Prism than the Light which went to the red end of that image, which proves as well the first proposition as the second.

Therefore, as in his New Theory, Newton arranges a sequence of experiments that starts with an experiment revealing a surprising effect to be explained, then proceeds to experiments that eliminate possible causal explanations before presenting his experimentum crucis as the one that positively and directly answers the question concerning the cause of the surprising effect on the basis of a difference test, which is the paradigmatic experiment for the inference of causal relations. This does not mean that Newton's experimentum crucis may not also be used to eliminate alternative explanations, such as modificationism. However, Newton does so in neither his New Theory nor his Opticks. Here, the experimentum crucis serves the purpose of providing the positive evidence needed to infer the cause by (noneliminative) induction.

It is not the individual experiments following proposition two, or even proposition one, that prove either of these propositions; instead, it is the experimentum crucis at the center of the sequence of experiments following these first two propositions that serves this purpose.

\section{Conclusion}

Newton never maintained that his experimental proofs were incompatible with alternative explanations for the observed experimental phenomena. However, possible alternatives create gaps in a proof only when a deductive axiomatic proof is taken as the benchmark. Instead, Newton's benchmark is whether a theorem can be proven from nothing but experimental evidence according to principles of induction. According to this measure, Newton's proof method used in his proof from the experimentum crucis is free of gaps because the principle of causality allows one to infer that properties of the light rays cause the refraction at the second prism and his second rule of reasoning in experimental philosophy allows one to generalize this inference to the refraction of the rays of sunlight at the first prism.

One might reject his proof by questioning the assumption of homogeneity. However, this is not a rejection of Newton's method itself but rather a rejection of the specific experimental conditions. Newton never questioned the relevance of such a critique (Turnbull, 1960, p. 79-80). One might also reject Newton's experimental proof because it is non-deductive. However, in doing so, one does not identify a gap within Newton's inductive experimental proof. According to Newton, a rejection of his proof method undermines experimental science because such a rejection does not allow for specific rules that ascribe probative force to experiments alone, without feigning hypotheses; see (Turnbull, 1959, p. 96-97), and Newton's fourth regula in the third edition of his Principia (Newton, 1962). Making use of rules in terms of postulates to generate a source of evidence and rules to identify decision criteria related to it (such as Euclidian definitions and common notions, 
Venn's rules to decide logical validity by his diagrams, or Newton's inductive rules to draw conclusions from experiments) has its price in being non-deductive. Yet, it solves the problem of being in need of a further justification as it is the case in starting from axioms or hypotheses and in making use of deductive rules, which have to be justified meta-logically in going beyond the pure calculus.

Like the analysis of Euclidean proofs and proofs in logic, the analysis of Newton's experimental proofs shows that only the iconic conception of proof can explain the probative force of these proofs. The reason is that the constituents of iconic proofs provide criteria for identifying relevant properties, whereas symbolic proofs consist of sentences from which one cannot read off relevant properties according to certain rules. Therefore, they lack the evidence that iconic proofs provide. Consequently, a claim that gaps exist in Euclid's or Newton's proofs shows that the interpreter has chosen the wrong conceptual framework in which to analyze the proofs in question; it does not identify deficiencies in the analyzed proofs themselves.

\section{REFERENCES}

Anstey, P., Hunter, M. (2008). Robert Boyle's 'Design about natural history', Early Science and Medicine 13, 83-126.

Beeson, M., Narboux, J., Wiedijk, F. (2019). Proofchecking Euclid, Annals of Mathematics and Artificial Intelligence 84(171), 1-45.

Byrne, O. (1847). The First Six Books of the Elements of Euclid in which coloured diagrams and symbols are used instead of letters for the greater ease of learners. London: William Pickering.

Cohen, I.B. (1958). Isaac Newton's papers and letters on natural philosophy and related documents. Harvard: Harvard University Press.

Dal Magro, T., García-Pérez, M.J. (2019). On Euclidean diagrams and geometrical knowledge, Theoria 34(2), 255-276.

Ducheyne, S. (2005). Newton's training in the Aristotelian textbook tradition: From effects to causes and back, History of Science 43, 217-236.

Giaquinto, M. (2011). Crossing curves: a limit to the use of diagrams in proofs, Philosophia Mathematica 19, 281-307.

Guerlac, H. (1973). Newton and the method of analysis. In Wiener (Ed.). Dictionary of the history of ideas 3 (pp. 378-391). New York: Scribners.

Guicciardini, N. (2016). Analysis and synthesis in Newton's mathematical work. In Cohen, B.I. \& Smith, G.E. (Eds.). The Cambridge Companion to Newton (pp. 308-38). Cambridge: Cambridge University Press.

Guicciardini, N. (2016). A brief introduction to the mathematical work of Isaac Newton. In Iliffe, R. \& Smith, G. (Eds.). The Cambridge companion to Newton (pp. 382-420). Cambridge: Cambridge University Press.

Hamou, P. (2018). Experimentum crucis. Newton's empiricism at the crossroads. In Bodenmann, S. \& Rey, A-L. (Eds.). What does it mean to be an empiricist? (pp. 47-69), New York et al.: Springer. Heath, T.L. (1968). The thirteen books of Euclid's Elements I. Cambridge: Cambridge University Press. Hintikka, J., Remes, U. (1974). The method of analysis. Dordrecht: Reidel.

Hintikka, J., Remes, U. (1976). Ancient geometrical analysis and modern logic. In Cohen, R.S. \& Feyerabend, P.K. \& Wartofsky, M.W. (Eds.). Essays in memory of Imre Lakatos (pp. 253-276). Dordrecht: Reidel.

Hooke, R. (1665). Micrographia, London: Martyn and Allestry.

Hooke, R. (1705). A discourse of earthquakes. In Waller, R. (Ed.). The posthumous works of Dr. Robert Hooke (pp. 277-450). London: Smith and Walford. 
Ihmig, K.N. (2004). Die Bedeutung der Methoden der Analyse und Synthese für Newtons Programm der Mathematisierung der Natur. In Meixner, U. \& Newen, A. (Eds.). Philosophiegeschichte und logische Analyse 7 (pp. 91-119). Paderborn: Mentis.

Jalobeanu, D. (2014). Constructing natural historical facts. Baconian natural history in Newton's First paper on light and colours. In Biener, Z. \& Schliesser, E. (Eds.). Newton and Empiricism (pp. 39-65). Oxford: Oxford University Press.

Lampert, T. (2017). Wittgenstein's ab-notation: An iconic proof procedure, History and Philosophy of Logic $38.3,239-262$.

Lampert, T. (2018). Iconic logic and ideal diagrams: The Wittgensteinian approach. In Chapman, P. \& Stapleton, G. \& Moktefi, A. \& Perez-Kriz, S. \& Bellucci, F. (Eds). Diagrammatic representation and inference (pp. 624-639). Cham: Springer.

Lampert, T. (2020). Decidability and notation, Logique et Analyse 251, 365-386.

Manders, K. (2008). The Euclidean diagram. In Mancosu, P. (Ed.). The philosophy of mathematical practice (pp. 80-133). New York: Oxford University Press.

Menn, S. (2002). Plato and the method of analysis, Phronesis 47.3, 193-223.

Miller, N. (2001). A diagrammatic formal system for Euclidean geometry, Cornell: Cornell University Press.

Mumma, J. (2012). Constructive geometrical reasoning and diagrams, Synthese 186, 103-119.

Newton, I. (1671/2). A letter of Mr. Isaac Newton containing his new theory about Light and colours. Philosophical Transactions 80, 3075-3087.

Newton, I. (1730). Opticks: Or a treatise of the reflections, refractions, inflections and colours of light. 4th edition. London: Innys.

Newton, I. (1962). Mathematical principles of natural philosophy and his System of the world. Motte, A. \& Cajori, F. (Trans.). Berkeley: University of California Press.

Pappus of Alexandria (1986). Book 7 of the Collection, Part . Introduction, Text and Translation. Jones, A. (Ed., Transl.). New York: Springer.

Sabra, A.I. (1981). Theories of light from Descartes to Newton. Cambridge: Cambridge University Press.

Schwartz, D. (2017). Crucial instances and Francis Bacon's quest for certainty, The Journal of the International Society for the History of Philosophy of Science 7, 130-150.

Shapiro, A.E. (2004). Newton's 'Experimental Philosophy', Early Science and Medicine, 9(3), 185-217.

Shimojima, A. (1996). Operational constrains in diagrammatic reasoning. In Barwise, J. \& Allwein, G. (Eds.). Logical Reasoning with Diagrams (pp. 27-48). Oxford: Oxford University Press.

Stapleton, G., Jamnik, M., Shimojima, A. (2016). Effective representation of information: Generalizing free rides. Jamnik, M. \& Shimojima, A. (Eds.). Diagramatic representation and inference (pp. 296-299), Cham: Springer.

Stapleton, G., Jamnik, M., Shikojima, A. (2017). What makes an effective representation of information: A formal account of observational advantages, Journal of Logic, Language and Information 26(2), 143-177.

Tarski, A. (1959). What is elementary geometry? In Henkin, L.A. \& Suppes, F. \& Tarski, A. (Eds.). The axiomatic method. Amsterdam: North Holland.

Thompson, E. (1995). Colour vision. A study in cognitive science and the philosophy of perception. London: Routledge.

Turnbull, H. W. (1959). The correspondence of Isaac Newton. Volume . 66-675, Cambridge, UK: Cambridge University Press.

Turnbull, H. W. (1960). The correspondence of Isaac Newton. Volume 2. 676-687, Cambridge, UK: Cambridge University Press.

TIMM LAMPERT has been associate professor for philosophy at the Humboldt University Berlin since 2014. His recent reasearch focusses on proof theory, iconic logic and decision procedures in first-order logic.

Address: Department of Philosophy, Humboldt University Berlin, Unter den Linden 6, D-10099 Berlin. E-mail: lampertt@staff.hu-berlin.de. ORCID: https://orcid.org/0000-0003-3912-7933 